\title{
DUST-DRIVEN WINDS OF ROTATING CARBON STARS
}

\author{
ERNST A. DORFI AND SUSANNE HÖFNER \\ Institut für Astronomie der Universität Wien \\ Vienna, Austria
}

\begin{abstract}
A new mechanism is proposed to explain an asymmetric mass loss of carbon-rich AGB stars where slow stellar rotation modifies a wind due to the non-linear behavior of the dust formation process. This effect leads to a preferential mass loss with higher velocities in the equatorial plane and also provides a simple explanation for the widely-observed asymmetries in the shapes of planetary nebulae.
\end{abstract}

\section{Introduction}

Stellar rotation is a ubiquitous phenomenon at all stages of stellar evolution, but older single stars are expected to exhibit only small rotational velocities at the photosphere due to magnetic braking occurring during their evolution and due to an expansion of the outer layers (e.g. Slettebak 1970). However, a small number of fast-rotating AGB objects like V Hya (Barnbaum et al. 1995) seem to exist where the outer envelope is interacting with a close companion. In the case of a planetary system around an expanding AGB star it is plausible that these outer stellar layers also can accumulate some of the angular momentum of the orbiting planets. Hence, it may well be that a larger fraction of AGB stars than expected exhibit rotation of a few $\mathrm{km} \mathrm{s}^{-1}$ in their photospheric layers without showing evidence for binarity. Since most of these stars are variable and maintain a modulated stellar wind of about $15 \mathrm{~km} \mathrm{~s}^{-1}$, it will be difficult to detect photospheric rotational velocites of a few $\mathrm{km} \mathrm{s}^{-1}$.

Taking into account some average observational properties, we construct dust-driven winds of slowly rotating carbon-rich AGB stars where only tiny deviations from spherical symmetry are present in the extended atmospheres, allowing a quasi-spherical approximation of the stellar structure (e.g. Kippenhahn et al. 1970). However, these small asymmetries between the equator and pole are sufficient to introduce significant changes in the dust condensation process through non-linearities and in the subsequently 
TABLE 1. Parameters of Dust-Driven Winds of Rotating AGB Stars

\begin{tabular}{rrrrccc}
\hline $\begin{array}{c}L \\
{\left[L_{\odot}\right]}\end{array}$ & $\begin{array}{c}\varepsilon_{\mathrm{C}} / \varepsilon_{\mathrm{O}} \\
{[\mathrm{C} / \mathrm{O}]}\end{array}$ & $\begin{array}{c}\omega \\
{\left[\mathrm{s}^{-1}\right]}\end{array}$ & $\begin{array}{c}v \sin i \\
{\left[\mathrm{~km} \mathrm{~s}^{-1}\right]}\end{array}$ & $\begin{array}{c}\dot{M} \\
{\left[10^{-6} M_{\odot} \mathrm{yr}^{-1}\right]}\end{array}$ & $\dot{M}_{\mathrm{e}} / \dot{M}_{\mathrm{p}}$ & $u_{\infty, \mathrm{e}} / u_{\infty, \mathrm{p}}$ \\
\hline $10^{4}$ & 2.3 & $2 \times 10^{-8}$ & 6.9 & 1.38 & 25.8 & 3.4 \\
$10^{4}$ & 2.3 & $1.5 \times 10^{-8}$ & 5.1 & 0.74 & 7.1 & 2.0 \\
$10^{4}$ & 2.3 & $10^{-8}$ & 3.4 & 0.44 & 2.6 & 1.4 \\
$10^{4}$ & 2.3 & $5 \times 10^{-9}$ & 1.7 & 0.31 & 1.3 & 1.1 \\
& & & & & & \\
$1.2 \times 10^{4}$ & 2.0 & $1.5 \times 10^{-8}$ & 5.6 & 1.24 & 9.7 & 2.4 \\
$1.2 \times 10^{4}$ & 2.0 & $10^{-8}$ & 3.8 & 0.65 & 3.2 & 1.6 \\
$1.2 \times 10^{4}$ & 2.0 & $10^{-9}$ & 0.4 & 0.38 & 1.0 & 1.0 \\
\hline
\end{tabular}

developing dust-driven outflow. The inner boundary of our dust-driven winds can be obtained from simple models of slowly rotating stars (e.g. Tassoul 1978), and the inclusion of small rotation rates already modifies the effective gravity and the scale height of the atmosphere, as well as the radiative flux. To get a quantitative picture of the dust-driven winds of a rotating AGB star we have solved the time-dependent system of grey radiation hydrodynamics together with the equations governing the dust formation in carbon-rich atmospheres (cf. Höfner et al. 1995 and Dorfi \& Höfner 1996 for further references and details).

\section{Outflow Properties and Mass Loss of Rotating AGB Stars}

The process of dust formation depends strongly on the density and temperature stratification in an extended atmosphere where even small changes e.g. in the density at a given temperature - can result in a totally different behavior of the stellar wind. Any small stellar surface fluctuations transported outwards are amplified in the dust-forming region, and therefore it is very likely that clumps are formed in the wind.

Some properties of the rotating dust-driven winds are summarized in Table 1 where the last three columns state the total mass loss rate $\dot{M}$, the ratio of equatorial to polar mass loss $\dot{M}_{\mathrm{e}} / \dot{M}_{\mathrm{p}}$, and the ratio of equatorial to polar final velocity $u_{\infty, \mathrm{e}} / u_{\infty, \mathrm{p}}$, respectively. It is evident that an increase in rotational velocity results in larger total mass-loss rates. This mass loss becomes more concentrated towards the equatorial plane and the angular difference in the outflow velocities also increases. The quantitative value clearly depends on the stellar parameters determining the effective gravity in the atmospheric layers as well as on the amount of carbon available to condense into grains. The two blocks of models (cf. Table 1) illustrate that the mass loss of the more extended model $\left(R=540 R_{\odot}\right)$ with a higher 

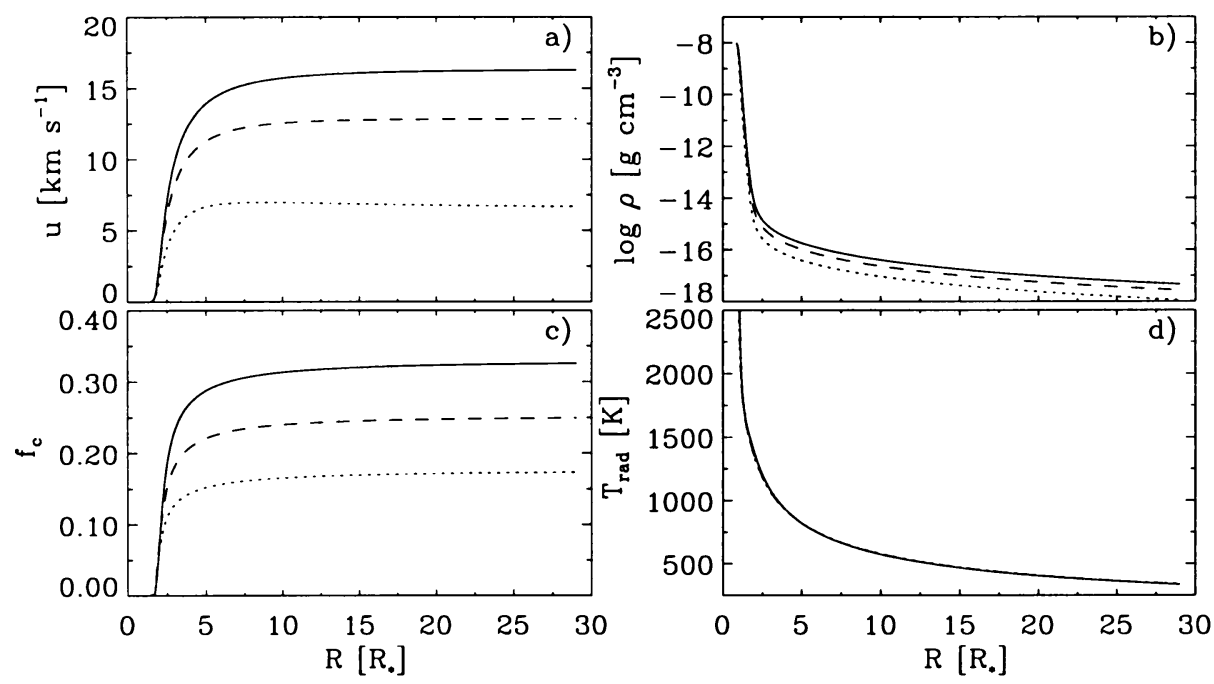

Figure 1. The radial structure of a rotating stationary dust-driven wind $\left(L=1.2 \times 10^{4}\right.$ $\left.L_{\odot}, M=1 M_{\odot}, T_{\text {eff }}=2600 \mathrm{~K}, \omega=1.5 \times 10^{-8} \mathrm{~s}^{-1}\right)$ plotted for three different angles: equatorial wind (solid line), $45^{\circ}$ (dashed line), and polar wind (dotted line); $(a)$ the gas velocity in units of $\mathrm{km} \mathrm{s}^{-1}$ between 1 and $30 R_{*} ;(b)$ the gas density; $(c)$ the degree of condensation of carbon dust grains; $(d)$ the radiation temperature.

luminosity of $L=1.2 \times 10^{4} L_{\odot}\left(M=1 M_{\odot}\right.$ and $\left.T_{\text {eff }}=2600 \mathrm{~K}\right)$ is more sensitive to the rotational velocity than the lower luminosity model $L=$ $10^{4} L_{\odot}$ corresponding to $R=493 R_{\odot}$. The stationary solutions obtained without rotation yield $\dot{M}=2.9 \times 10^{-7} M_{\odot} \mathrm{yr}^{-1}$ with $u_{\infty}=12 \mathrm{~km} \mathrm{~s}^{-1}$ and $\dot{M}=3.8 \times 10^{-7} M_{\odot} \mathrm{yr}^{-1}$ with $u_{\infty}=9.5 \mathrm{~km} \mathrm{~s}^{-1}$. In the stationary solutions discussed here the carbon-to-oxygen ratio $\varepsilon_{\mathrm{C}} / \varepsilon_{\mathrm{O}}$ has to be large enough to drive a purely dust-driven wind, whereas the time-dependent solutions can be obtained with much smaller values of $\varepsilon_{\mathrm{C}} / \varepsilon_{\mathrm{O}}$ (e.g. Höfner \& Dorfi 1997). In the latter case the stellar pulsations depost kinetic energy in the atmospheric layers, lifting more condensable material into the dust formation zone.

A typical spatial structure of a dust-driven wind generated by a rotating carbon-rich AGB star is plotted for different angles in Figure 1. The massloss rate, velocity, and degree of condensation $f_{\mathrm{c}}$ increase towards the equatorial plane (solid lines). Since the radiation fields depicted by the radiation temperature $T_{\text {rad }}$ in Fig. $1 d$ are almost identical, the angular wind variation is mainly caused by the difference in the gas density (cf. Fig. 1b) which can strongly amplify the dust condensation, controlling the outflow.

The mass loss as a function of the polar angle $\theta$ is plotted in Figure 2 where the lines correspond to a fit by $\dot{M}(\theta)=\dot{M}_{\mathrm{p}}\left(1+\varepsilon \sin ^{n} \theta\right)$. Kahn and West (1985) have assumed such a dependence of the mass-loss rate 


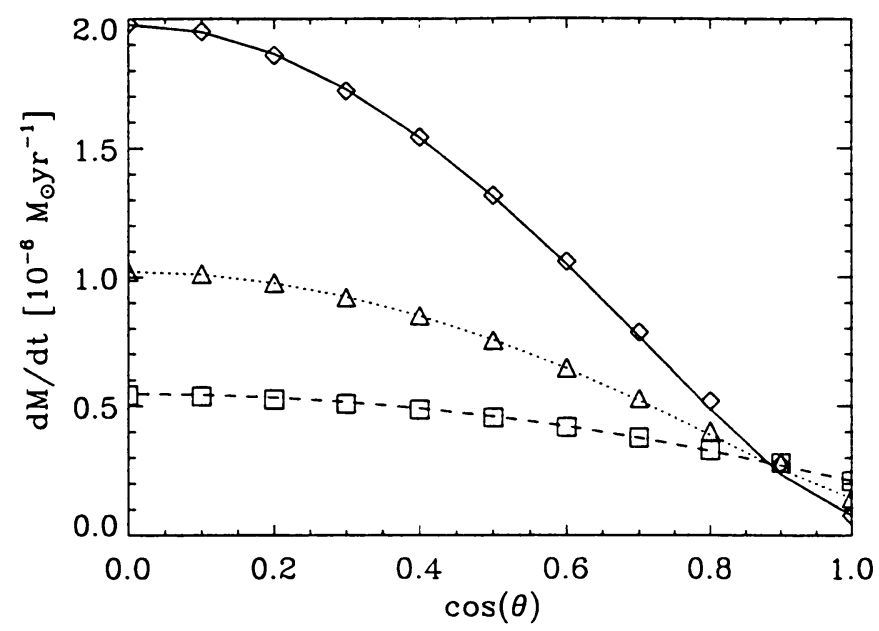

Figure 2. The mass-loss rates for different rotational velocities $\omega$ of an AGB star with $M=1 M_{\odot}, L=10^{4} L_{\odot}$ and $T_{\text {eff }}=2600 \mathrm{~K}$ (diamonds: $\omega=2 \times 10^{-8} \mathrm{~s}^{-1}$, triangles: $\omega=1.5 \times 10^{-8} \mathrm{~s}^{-1}$, squares: $\omega=10^{-8} \mathrm{~s}^{-1}$ ) as a function of the polar angle $\theta$. The equatorial plane is denoted by $\cos \theta=0$. The different lines are analytical fits according to Kahn \& West (1985).

on the angle $\theta$ to explain the shaping of planetary nebulae in their twowind model. The subsequently developing spherical wind of the white dwarf blows into the angle-dependent, slowly outflowing material of the former red giant. Our calculations yield values of $n \simeq 2 \ldots 3$ where $n=2$ is valid for the slowest angular velocities, i.e. $\omega=10^{-9} \mathrm{~s}^{-1}$. Most of the variation of the wind as a function of the rotation period is contained in the second parameter and given by $\varepsilon=\dot{M}_{\mathrm{e}} / \dot{M}_{\mathrm{p}}-1$. So far we have not obtained a simple relation to estimate the polar mass loss rate $\dot{M}_{\mathrm{p}}$, the exponent $n$ and the ratio $\dot{M}_{\mathrm{e}} / \dot{M}_{\mathrm{p}}$ as a function of the fundamental stellar parameters. The overall mass loss of a star evolving along the AGB will also depend on the angular momentum distribution inside the stellar mass zones which gradually become the stellar surface when the star loses more and more of its mass. Without having detailed stellar models of rotating AGB stars, it is therefore impossible to calculate the exact shaping of planetary nebulae based on such a two-wind model.

\section{Discussion}

The stellar evolution on the AGB (e.g. Iben \& Renzini 1983; Lattanzio 1986) complicates this simplfied picture of a dust-driven wind in several ways. First, as the star produces its heavier elements by thermonuclear reactions in the interior, the chemical composition of the outer layers changes due to several dredge-up events, and accordingly the condensation process 
of dust particles yields different grains which are then accelerated by a luminosity which increases during the AGB phase. Second, in the case of faster rotations than, for example, taking the solar rotation rate and expanding the star to AGB radii while conserving angular momentum, we run into the problem that the mass-loss rate depends strongly on the polar angle. There exists a lower limit for dust-driven stationary winds (Gail \& Sedlmayr 1987), and in the fastest rotating models of Table 1 the polar mass-loss rate of about $\dot{M}_{\mathrm{p}} \simeq 5 \times 10^{-8} M_{\odot} \mathrm{yr}^{-1}$ is already close to this limit. In such cases the nature of the polar wind must be different and cannot be supported by the radiation pressure on newly formed dust grains. Third, the stationary solutions presented are possible only in a limited parameter range due to the so-called dust-induced $\kappa$-mechanism leading to non-stationary dustdriven outflows (Fleischer et al. 1995; Höfner et al. 1995). This instability is more likely to start in the denser equatorial regions, whereas the polar wind is still maintained by a stationary wind. Fourth, the overall flow structure can contain a number of clumps since the time scale for dust condensation is very sensitive to density fluctuations and varies for different polar angles. Furthermore, it is not clear how the angular momentum is redistributed in a rotating star having a preferential mass loss (and therefore clumps) in the equatorial region. If the rotation rate decreases as more and more atmospheric layers are blown away, the overall mass loss becomes more spherical and further surface fluctuations may allow the formation of clumps also at higher latitudes. Fifth, in calculating our dust-driven models we have assumed the most conservative case of keeping the angular momentum constant within the expanding flow. If weak magnetic fields permeate the stellar atmosphere and the dust formation zone, causing a slower decrease of the centrifugal acceleration than $a_{\omega} \propto r^{-3}$ by angular momentum transfer, the effect of preferential mass loss towards the equatorial plane is strongly enhanced. However, to quantify this statement the exact topology of the magnetic field has to be known.

\section{Conclusions}

On the basis of a dust-driven wind generated by a slowly rotating carbonrich red giant (e.g. $T_{\text {eff }}=2600 \mathrm{~K}, M=1 M_{\odot}, L=10^{4} L_{\odot}$ ) with a photospheric rotational velocity of about $2 \mathrm{~km} \mathrm{~s}^{-1}$, we obtain a total mass loss increased by at least a factor of 1.5 relative to the mass loss of the nonrotating star. The mass loss in the equatorial plane is about 2 times larger than in the polar direction and is accompanied by an increase of the terminal velocity in going from the pole towards the equator. Note that these changes are due to the non-linear modifications of the dust formation process introduced by small deviations from spherical symmetry. Such an asymmetric mass loss during the AGB phase could explain the bipolar 
appearance of many planetary nebulae according to interacting wind models (e.g. Kwok 1982; Kahn \& West 1985). A density contrast of a factor of 2 can easily be generated by a very small stellar rotation and is already sufficient to produce a bipolar structure. Since this proposed new mechanism is by no means restricted to time-independent solutions, we are currently investigating time-dependent models of dust-driven winds of pulsating and slowly rotating AGB stars.

This work is supported by the Österreichischer Fonds zur Förderung der wissenschaftlichen Forschung (FWF) under project number S7305-AST.

\section{References}

Barnbaum, C., Morris, M. \& Kahane, C. 1995, ApJ, 450, 862

Dorfi, E. A. \& Höfner, S. 1996, $A \& A, 313,605$

Fleischer, A. J., Gauger, A. \& Sedlmayr, E. 1995, $A \& A, 297,543$

Gail, H.-P. \& Sedlmayr, E. 1987, $A \& A, 177,186$

Höfner, S. \& Dorfi, E.A. 1997, $A \& A, 319,648$

Höfner, S., Feuchtinger, M. U. \& Dorfi, E.A. 1995, $A \& A, 297,815$

Iben, I. Jr. \& Renzini, A. 1983, Ann. Rev. Astron. Astrophys., 21, 271

Kahn, F.D. and West, K.A. 1985, MNRAS, 212, 837

Kippenhahn, R., Meyer-Hofmeister, E. \& Thomas H. C. 1970, $A \& A, 5,155$

Kwok, S. 1982, ApJ, 258, 280

Lattanzio, J.C. 1986, ApJ, 311, 708

Slettebak, A. (ed.) 1970, IAU Coll. on Stellar Rotation (D. Reidel)

Tassoul, J.-L. 1978, Theory of Rotating Stars (Princeton Univ. Press)

\section{Discussion}

Cherchneff: How do you describe the dust formation process in your model? Is it the same description at the pole and at the equator?

Dorfi: We take the dust formation and growth formalism developed by Gail \& Sedlmayr and Gauger et al. (1988) to calculate the dust-driven winds. We used the same description for the polar and equatorial regions.

Jorissen: Would your model be able to account for the fast outflow $(\sim 200$ $\mathrm{km} \mathrm{s}^{-1}$ ) observed in the rotating $\left(v \sin i \approx 15 \mathrm{~km} \mathrm{~s}^{-1}\right)$ carbon star V Hya?

Dorfi: In the case of $\mathrm{V}$ Hya, I expect from our model a very asymmetric wind driven by condensation of $\mathrm{C}$ grains. However it will be very difficult to drive the polar wind since the mass-loss rate goes down in the polar regions. Hence I think that a mechanism other than radiation pressure on $\mathrm{C}$ grains is responsible for this high-velocity outflow.

Plez: The fast wind of V Hya was first detected by T. Lloyd Evans through observation of Fe II emission lines. Long-slit spectroscopy of the K I $7699 \AA$ line by D.L. Lambert and myself shows the bipolar structure of the fast component at about $150 \mathrm{~km} \mathrm{~s}^{-1}$ (Plez \& Lambert 1994, ApJ, 425, L101). 